\title{
Modeling and Mitigating Transient Errors in Logic Circuits
}

\author{
Ilia Polian, Senior Member, IEEE, John P. Hayes, Fellow, IEEE, \\ Sudhakar M. Reddy, Fellow, IEEE, and Bernd Becker, Fellow, IEEE
}

\begin{abstract}
Transient or soft errors caused by various environmental effects are a growing concern in micro and nanoelectronics. We present a general framework for modeling and mitigating the logical effects of such errors in digital circuits. We observe that some errors have time-bounded effects; the system's output is corrupted for a few clock cycles, after which it recovers automatically. Since such erroneous behavior can be tolerated by some applications, i.e., it is noncritical at the system level, we define the critical soft error rate (CSER) as a more realistic alternative to the conventional SER measure. A simplified technology-independent fault model, the single transient fault (STF), is proposed for efficiently estimating the error probabilities associated with individual nodes in both combinational and sequential logic. STFs can be used to compute various other useful metrics for the faults and errors of interest, and the required computations can leverage the large body of existing methods and tools designed for (permanent) stuck-at faults. As an application of the proposed methodology, we introduce a systematic strategy for hardening logic circuits against transient faults. The goal is to achieve a desired level of CSER at minimum cost by selecting a subset of nodes for hardening against STFs. Exact and approximate algorithms to solve the node selection problem are presented. The effectiveness of this approach is demonstrated by experiments with the ISCAS-85 and -89 benchmark suites, as well as some large (multimillion-gate) industrial circuits.
\end{abstract}

Index Terms-Soft errors, error tolerance, selective hardening, transient faults.

\section{INTRODUCTION}

$\mathrm{T}$ RANSIENT or soft errors are temporary deviations of a circuit's behavior from its correct or reference behavior. They are caused by single-event upsets (SEUs) due to particle strikes, electrical noise, or other environmental effects, and are a major concern in advanced digital ICs [25], [3]. They occur at unpredictable times, and so require probabilistic methods to analyze their effects or to synthesize circuits that mitigate their impact. Most approaches to these issues tend to be heuristic, and employ models that are technology or application-dependent and computationally complex. Existing methods of guarding against soft errors rely on large amounts of redundancy and incur significant overhead costs. This is particularly true for logic circuits, where techniques like ECC that are effective for memories are not applicable.

In many applications, transient errors are acceptable as long as the correct behavior is restored quickly. Suppose, for example, a system relies on input data from unreliable sensors. It must work properly even if a sensor occasionally fails to deliver its data. Such a transient fault can deteriorate

- I. Polian is with the University of Passau, Innstr. 43, D-94032, Passau, Germany. E-mail: polian@informatik.uni-freiburg.de.

- J.P. Hayes is with the Department of Electrical Engineering and Computer Science, University of Michigan, 2260 Hayward Street, Ann Arbor, MI 48109-2122.E-mail: jhayes@eecs.umich.edu.

- S.M. Reddy is with the ECE Department, The University of Iowa, 5324 Seamans Center for the Engineering Arts and Sciences, Iowa City, IA 52242. E-mail: sudhakar-reddy@uiowa.edu.

- B. Becker is with the University of Freiburg, Georges-Koehler-Allee 51, D-79110, Freiburg, Germany. E-mail: becker@informatik.uni-freiburg.de.

Manuscript received 12 Dec. 2008; revised 10 Dec. 2009; accepted 22 Jan. 2010; published online 2 Aug. 2010.

For information on obtaining reprints of this article, please send e-mail to: tdsc@computer.org, and reference IEEECS Log Number TDSC-2008-12-0193. Digital Object Identifier no. 10.1109/2010.26. the system's output for a few clock cycles, after which it returns to error-free operations without any recovery efforts. Further examples are video systems that tolerate a few missing pixels [18], network applications that handle errors by a retransmission [21], and data processing units implementing commit rollback recovery [9]. In embedded systems with a human end-user, brief deviations of the output data from their correct values may not be perceptible, and therefore, are easily tolerated [5].

In this work, we explore the modeling of transient faults and errors, and the computation of their occurrence probabilities. This is a challenge because soft errors occur at random times, and their impact is highly dependent on circuit's state. To capture the faults of interest, we introduce the single transient fault (STF) model, which makes it possible to efficiently estimate error probabilities in logic circuits. STFs can also be used to compute other useful metrics, and they can be evaluated by means of existing methods and software tools designed for (permanent) stuck-at faults.

We also explore the issue of transient-error tolerance (TET), which is based on the observation that not all errors at a circuit's output are equally critical. A transient error is considered noncritical or tolerable if it disappears within a specified time, the noncritical error period (NEP), with some specified probability. Noncritical soft errors can be excluded from the SER resulting in a new and more realistic metric called the critical SER (CSER). No protection is needed against noncritical errors, thus potentially reducing design costs. To illustrate the proposed methodology, we present a technique for selective hardening of nodes to maximize the probability of error-tolerant operation, measured by a metric called derating factor. We study the tradeoffs between hardening cost and CSER reduction, using the STF model as a vehicle. 
The rest of the paper is organized as follows: the STF model is introduced in Section 2. The concept of transienterror tolerance is defined in Section 3, and its application to combinational and sequential circuits is discussed in Sections 4 and 5, respectively. The notion of CSER is introduced in Section 6. Exact and approximate algorithms for selective hardening of circuits are described in Section 7. Experimental results are reported in Section 8. Section 9 concludes the paper.

\section{Single transient fault Model}

Early research on soft errors addressed various behavioral and statistical aspects of intermittent and transient faults without defining explicit fault models for them [6], [32]. Soft error modeling has recently received significant attention. There are two broad areas in which advances have been made: low-level modeling and high-level modeling. Lowlevel approaches [7], [12], [39] aim at exact modeling of physical processes which take place when an energetic particle hits a device in a CMOS digital circuit. One issue of particular interest is the creation of a voltage glitch following a particle strike at a $p n$ junction within a memory cell, a flip-flop, or a logic gate. Another aspect is the propagation of the glitch through the combinational logic to an observable point of the system, e.g., a flip-flop. The glitch may not result in any visible effect due to three masking mechanisms: logical masking, latching-window masking, and electrical masking [35].

High-level approaches are concerned with the soft errors that actually affect the system behavior (i.e., are not masked) [8], [23], [19]. One key question is whether the protection mechanisms that the system provides are sufficient to keep the consequences of a soft error in check. High-level methods typically model a soft error as a bit-flip in a memory location, in a state element (flip-flop), or at a logic line of the circuit, with a duration of one clock cycle.

While different low-level models have varying degrees of accuracy, they usually agree on two issues. First, particle strikes of sufficient energy to cause a bit-flip are still relatively rare in ground-level applications, and this is expected to stay so for the near future. Hence, the probability of multiple particle strikes within a short period or even within the same clock cycle is assumed to be negligible. Second, the duration of most particle-induced glitches is less than the cycle time even for high-speed circuits. Although diffusion processes may still be transporting charge, i.e., electrons or holes ionized by particle impact, to a $p n$ junction for a relatively long time, the amount of charge carriers is not sufficient to cause bit-flips. Thus, the immediate effect of one particle strike is confined to the clock cycle in which it occurs. Multicycle effects require that an erroneous logical value be stored in a flip-flop and the state of the circuit is corrupted.

The STF model used in this paper belongs to the class of high-level models. Its focus is on the effects of a particle strike on system behavior. It is not intended to accurately represent low-level details of particle-strike physics or glitch propagation; other (electrical) models exist for this purpose. In this respect, the STF model is fully consistent with standard testing philosophy where relatively simple

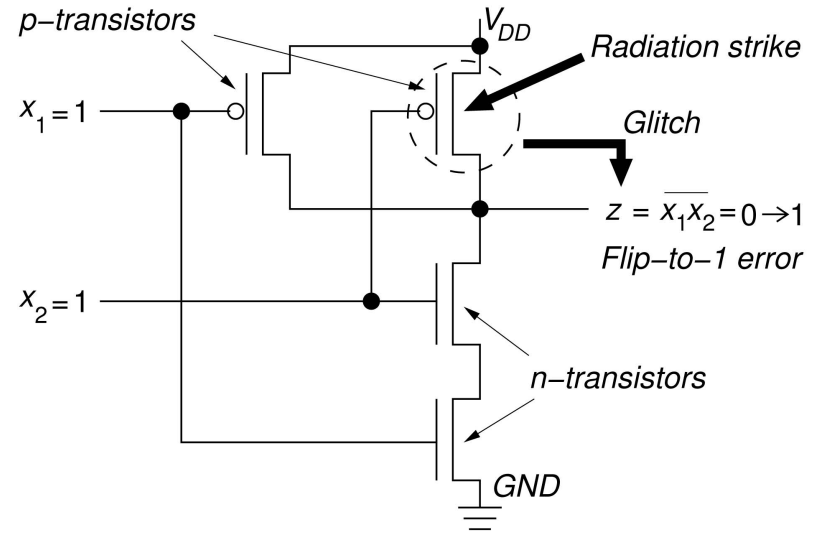

Fig. 1. A NAND2 gate affected by a transient flip-to-1 error.

fault models such as stuck-at faults, four-way bridging faults, and transition faults are used almost universally for test pattern generation, fault simulation, and related tasks.

The target circuits are assumed to be synchronous logic circuits composed of gates, flip-flops, RTL elements, etc. Let $C=(I, O, S, \delta, \lambda)$ denote a sequential circuit (finite-state machine) with $k$ logic lines. Here, $I$ is the input alphabet (the set of input values), $O$ is the output alphabet, $S$ is the set of internal states, $\delta$ is the next-state function, and $\lambda$ is the output function. A single transient fault in $C$, denoted by $f(l / p, x, s)$, is defined by the following properties: 1$)$ it causes the line $l$ to be stuck-at $p$, where $p$ is 0 or 1 , for one clock cycle, and 2) the associated total state of $C$ is $x, s$, where $x \in I$ and $s \in S$. The number of distinct STFs in $C$ is $2 k|I||S|$, where $|I|$ and $|S|$ are the cardinalities of $I$ and $S$, respectively. While this number is large, it is not intractable if implicit techniques are used (see below). Often, we can restrict attention to small or easily computed classes of STFs.

Observe that there is no cause-effect relationship between an STF and its associated state; $f(l / p, x, s)$ is an STF that occurs when $C$ is in state $x, s$. The STF model is clearly related to the standard stuck-at fault (SAF) model. Unlike an $\mathrm{STF}$, an SAF $l / p$ persists indefinitely once it occurs and is not associated with specific states. Many simulation and ATPG tools for SAFs can readily be applied to STFs.

More complex transient faults can be modeled by probability transfer matrices (PTMs) [17] which, however, require linear algebra rather than Boolean algebra for their analysis. PTMs also tend to be memory bound. Moreover, it is generally possible to enrich the STF model by low-level data, for example, to assign each gate and each input combination a specific susceptibility. Consider the twoinput CMOS gate NAND2 in Fig. 1. A radiation strike can upset one or more of its transistors, causing the output $z$ to undergo a transient flip-to-0 or flip-to- 1 error. The specific error depends in part on the input pattern $x_{1} x_{2}$ when the strike occurs.

Input $x_{1} x_{2}=11$ flips $z$ from 0 to 1 if one of the gate's $p$ transistors is upset, as in Fig. 1. Under input patterns 10 and 01 , only one $n$-transistor is susceptible to the strike. With input 00, both $n$-transistors must be upset to produce an output bit-flip. The probability that a transistor or a combination of transistors is upset by a particle can be calculated using an SER analysis tool such as TMC-DASIE [31] which is based on accurate modeling 
of nuclear reactions. Based on these data, soft error susceptibility or the upset probability of the NAND2 gate under inputs $00,01,10$, and 11 can be set to some values $p_{00}, p_{01}, p_{10}$, and $p_{11}$, respectively.

\section{Transient-Error Tolerance}

An error that finds its way into the internal state may be eliminated by suitable design methods, or it may simply be flushed out automatically by normal inputs that happen to take the circuit to a correct state. The probability of such selfrecovery is of interest. A circuit $C$ is transient-error-tolerant for the STF set $F$ with noncritical error period $k$ and self-recovery probability $p_{s r}$, denoted by $\left(F, k, p_{s r}\right)$-TET, if the internal states of the erroneous and error-free circuits are the same after $k$ cycles with the probability of at least $p_{s r}$, assuming equiprobable inputs. $C$ is $\left(k, p_{s r}\right)$-TET if the conditional probability that its state is error-free $k$ cycles after an arbitrary STF occurs is at least $p_{s r}$.

For $p_{s r}=1.0$, the circuit $C$ is $(F, k, 1.0)$-TET, if the state of $C$ affected by any member of $F$ and that of the error-free circuit are the same after $k$ clock cycles for all possible input sequences. Note that the initial state is implicitly included in each fault $f$ of $F$. A combinational circuit is thus always $(1,1.0)$-TET since it recovers from an STF after one clock cycle. A sequential circuit is $(1,1.0)$-TET for all faults that influence only its primary outputs, but not its next state (memory) part. A feedback-free pipelined circuit of depth $m$ is $(m, 1.0)$-TET for all STFs. It has been shown for a motion estimation circuit that it is TET with $p_{s r}=1.0$ for over 70 percent of its faults with period 96 [28]. This means that if one of these faults occurs, the encoding of the image being processed may be suboptimal. However, the encoding of subsequent images, starting with the next image completely transmitted, will be performed as if the fault has never occurred.

Further error-tolerance concepts of interest include error significance and error rate. Error significance denotes the impact of an error from an application point of view [5], [15]. For instance, peak signal-to-noise-ratio, structural similarity, and psychovisual deviation are used as error significance metrics for a JPEG encoder in [26]. The notion of error rate defines errors with a sufficiently low probability of occurrence as noncritical [34]. A metric called SBER, which combines error rate and error significance, has been proposed in [27]. Error significance and error rate have mostly been studied for permanent faults although they can be readily applied to soft errors.

Another class of potentially noncritical errors affects performance-enhancing units such as branch predictors in microprocessors [2]. These errors do not prevent the calculation of the correct results but delay (time-shift) its completion. Similar effects can also be observed for errors handled by a commit-rollback-recovery scheme [9]. Noncritical error effects have also been studied in the context of real-time systems under the heading of imprecise computation [20].

\section{Errors in Combinational Logic}

Since there is no internal state $s$, an STF for a combinational circuit $C$ reduces to the form $f(l / p, x)$. An STF error then corresponds to SAF $l / p$ and a test $x$ for $l / p$, since by

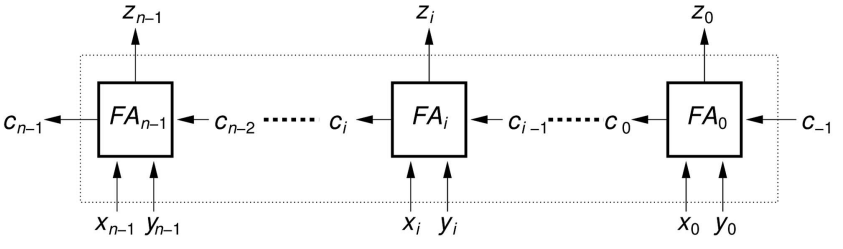

Fig. 2. An $n$-bit ripple-carry adder.

definition, a test propagates the fault effect (error) to a primary output. Let $C$ have $k$ lines, $n$ primary inputs, and a single primary output $z$, and assume that all STFs are equiprobable. The STF error probability $p_{\text {err }}(z)$ is the total number of possible errors produced at $z$ by STFs divided by the total number of possible STFs as follows:

$$
p_{\text {err }}(z)=\frac{\sum_{l} \text { No. of tests for the faulty line } l}{k 2^{n+1}} .
$$

Suppose $C$ is an $n$-input gate of the (N)AND or (N)OR type. Equation (1) implies that

$$
p_{\text {err }}(z)=\left(n+2^{n-1}\right) /(n+1) 2^{n} .
$$

Here, $p_{\text {err }}(z)$ approaches $1 /(2(n+1))=1 /(2 k)$ as $n$ increases, which means that gates with greater fan-in are more likely to mask or tolerate STF errors. In the case of a gate of the $\mathrm{X}(\mathrm{N}) \mathrm{OR}$ type, $p_{\text {err }}(z)=1 / 2$. In general, the STF $f(l / p, x)$ is only detectable by input vectors that make $l=\bar{p}$; it is undetectable if $l=p$. Gates of the (N)AND/(N)OR type and gates of the $\mathrm{X}(\mathrm{N}) \mathrm{OR}$ type are therefore the best and worst cases, respectively, in terms of error masking among all $n$-input logic functions. We conclude that for any $k$-line single-output combinational circuit

$$
1 /(2 k) \leq p_{\text {err }}(z) \leq 1 / 2 .
$$

Hence, STFs capture our intuitive notions of transient error propagation and logical masking quite well. Note that electrical and latching window masking [36] are technologydependent and not included in our model. As will be discussed in Section 7.2, recent experimental data [40] suggest that these masking mechanisms have little influence when selecting circuit nodes for hardening.

\subsection{Ripple-Carry Adder}

Consider the $n$-bit ripple-carry (RC) adder of Fig. 2 . It is constructed from a full adder $F A_{i}$, an RTL element realizing two functions, the sum $z_{i}$, and the carry-out $c_{i}$. It has $n F A_{i}$ stages, $2 n+1$ inputs, and $n+1$ outputs. There are $4 n+1$ lines that can be faulty, so the total number of STFs is $(4 n+1) 2^{2 n+2}$.

We can compute the output error probabilities $p_{\text {err }}\left(z_{i}\right)$ by counting the errors produced at $z_{i}$ by STFs associated with a representative element $F A_{i}$. We can also subdivide the errors on $z_{i}$ and $c_{i}$ into two groups as follows: local errors due to faults in $F A_{i}$ itself, and remote errors that originate in preceding stages, and enter $F A_{i}$ via its carry-in line $c_{i-1}$. The local error count at $z_{i}$ is $2^{2 n+3}$ and the corresponding remote error count is $e\left(c_{i}\right)=2^{2 n+2}+2 e\left(c_{i-1}\right)$, leading to the following formula for the STF error probability on $z_{i}$ : 
TABLE 1

STF Error Probabilities in RC Adders

\begin{tabular}{c|c|c|c|c|c|c|c|c|c} 
Size $n$ & Carry $c_{n-1}$ & $z_{0}$ & $z_{1}$ & $z_{2}$ & $z_{3}$ & $z_{4}$ & $z_{5}$ & $z_{6}$ & $z_{7}$ \\
\hline 1 & 0.250 & 0.400 & & & & & & & \\
2 & 0.181 & 0.222 & 0.306 & & & & & & \\
4 & 0.112 & 0.118 & 0.282 & 0.185 & 0.195 & & & & \\
6 & 0.079 & 0.080 & 0.110 & 0.125 & 0.132 & 0.136 & 0.138 & & \\
8 & 0.060 & 0.060 & 0.083 & 0.095 & 0.100 & 0.103 & 0.105 & 0.105 & 0.106
\end{tabular}

$$
p_{\text {err }}\left(z_{i}\right)=\frac{2^{2 n+3}+2^{2(n-i)}\left(e\left(c_{i-1}\right)-2^{2 i+1}\right)}{(4 n+1) 2^{2 n+2}} .
$$

Table 1 shows some $p_{\text {err }}$ values derived from this analysis. Such data provide useful information about a circuit's error propagation or masking properties. For example, the $p_{\text {err }}\left(z_{i}\right)$ s of the RC adder increase slowly with $i$, eventually leveling off. The error probability $p_{e r r}\left(c_{n-1}\right)$ at the final carry-out is always less than that of the $z_{i}$ (sum) outputs.

\subsection{Exact Calculation of $p_{\text {err }}$}

Consider a general, $n$-input combinational circuit $C$ with $m$ output functions $Z=z_{1}, z_{2}, \ldots, z_{m}$. The calculation of the STF error probability $p_{\text {err }}\left(z_{i}\right)$ of the output $z_{i}$ according to (1) can be expressed in terms of the detection probabilities of stuck-at faults as follows:

$$
p_{\text {err }}\left(z_{i}\right)=\frac{\sum_{f \in S A F} D P\left(f, z_{i}\right)}{|S A F|},
$$

where $S A F$ is the set of all the stuck-at faults, $|S A F|$ is the size of this set, and

$$
D P\left(f, z_{i}\right)=\left|z_{i} \oplus z_{i}^{f}\right| / 2^{n}
$$

is the detection probability of stuck-at fault $f$ at the output $z_{i}$. On the right-hand side of (6), $z_{i}$ is the function of the circuit $C^{\prime}$ s $i$ th output, and $z_{i}^{f}$ is the same function with fault $f$ present. The function $z_{i} \oplus z_{i}^{f}$ maps all input vectors for which the fault-free and the faulty circuits calculate different values to 1 and all other input vectors to 0 . The term $\left|z_{i} \oplus z_{i}^{f}\right|$ denotes the cardinality of this function's on-set, i.e., the number of input vectors mapped to 1 , and thus, the number of all input vectors for which fault $f$ is detected at the output $z_{i}$. Each stuck-at fault in (5) subsumes $2^{n}$ STFs: an STF $f(l / p, x)$ is represented by the $l$ stuck-at- $p$ fault. Note that the stuck-at faults are only used as a means for efficient computation of $p_{e r r}\left(z_{i}\right)$; the physical disturbances modeled by STFs (transient errors) and stuck-at faults (permanent defects) are entirely different.

The circuit $C^{\prime}$ s error probability $p_{e r r}(C)$ considering all $m$ outputs is expressed by

$$
p_{\text {err }}(C)=\frac{\sum_{f \in S A F} D P(f)}{|S A F|},
$$

with

$$
D P(f)=\left|\bigvee_{i=1}^{m}\left(z_{i} \oplus z_{i}^{f}\right)\right| / 2^{n} .
$$

Equations (7) and (8) can be evaluated efficiently using symbolic simulation with binary decision diagrams (BDDs) representing functions $z_{i}$ and $z_{i}^{f}$. They can also be approximated using random-pattern simulation techniques from [4] or the methods described in the next section.

\subsection{Approximate Calculation of $p_{\text {err }}$}

While exact calculation of detection probabilities (8) is an $\mathrm{NP}$-complete problem, it is possible to compute the values of $D P(f)$ by a fast (linear in the size of the circuit) heuristic. These approximate detection probability values can be used in (7) for circuits for which BDDs cannot be constructed. Obviously, the resulting $p_{\text {err }}$ values are approximations of the exact values.

In this work, approximate calculation of detection probabilities is performed in a way similar to STAFAN [14] and PROTEST [37]. Two passes through the circuit are required. In the first pass of the algorithm, the circuit is traversed in topological order (from inputs to outputs). The signal probabilities of all lines in the circuit are determined by assigning a signal probability of 0.5 to each primary input and deriving the signal probability on the output of a gate from those on the gate's inputs. In the second pass, the circuit is traversed in reverse topological order (from outputs to inputs). The detection probabilities of faults on the circuit's outputs are computed from their signal probabilities. Then, detection probabilities of all other lines (inputs of some gate $g$ ) are determined from the detection probability of $g^{\prime}$ s output and the signal probabilities on $g^{\prime}$ s side-inputs. Details of the algorithm can be found in [30].

\section{Errors in Seguential Circuits}

We illustrate the foregoing TET concepts and formalisms using a small serial adder (SA) circuit with just two states for which a Markov model is easily constructed. We then propose a general method to calculate TET properties for sequential circuits. Experimental results for ISCAS-89 circuits can be found in Section 8.3.

\subsection{Serial Adder}

Consider the serial adder in Fig. 3. This sequential circuit adds two binary numbers $X$ and $Y$ serially (bit by bit) to produce the sum $Z$. It comprises a combinational adder $F A$, a D flip-flop $D F F$ which stores the carry bit $c$, and, counting only the lines visible in Fig. 3, a total of 80 STFs.

Now consider the effect of an STF $f$ on the output $z$ and the next state $c$ in the initial clock cycle 0 when the STF occurs. Table 2 places each STF $f$ into one of four sets based on whether or not $f$ produces an erroneous value of $z$ and/ or $c$ in clock cycle 0 . As implied by (3), half the possible STFs are undetectable, so SA is $\left(F_{0}, 0,1.0\right)$-TET. Class $F_{1}$ 


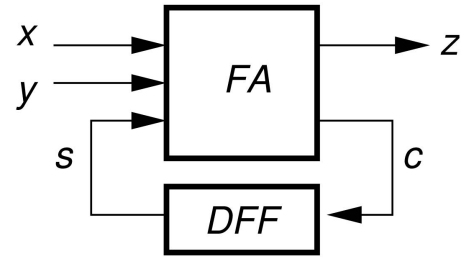

Fig. 3. RTL model of a serial adder SA.

represents the case where SA's output, but not its internal state, is erroneous; hence SA is $\left(F_{01}, 1,1.0\right)$-TET, where $F_{01}=F_{0} \cup F_{1}$. Thus, if an error is acceptable at $z$ in cycles 0 and 1 only, i.e., the NEP $k=1$, then all STFs in $F_{01}$ are tolerated. Since these represent 75 percent of the possible STFs, we can say that SA is $(1,0.75)$-TET. SA is also $(1,0.875)$-TET, because although $F_{2}$ and $F_{3}$ can produce error states in cycle 1 and beyond, the probability of them doing so is 0.5 , as will become clear later, and the share of $F_{2}$ and $F_{3}$ is 0.25 yielding $0.75+0.5 \cdot 0.25=0.875$.

It is easily seen that the STFs in Table 2 include a few faults that can leave an error lurking indefinitely in the circuit's internal state. Thus SA is not $(k, 1.0)$-TET for any finite $k$ when all STFs are considered.

Self-recovery can be analyzed by Markov models [6]. We use them to compute the probability $p_{\text {good }}$ of the circuit going from erroneous states induced by STFs to correct states within $k$ clock cycles. We can then say that the circuit is $\left(k, p_{\text {good }}\right)$-TET.

Considering SA again, its state transition graph is in Fig. 4a. For half the input patterns $x y$, the next internal state $c$ (but not the output $z$ ) is independent of the initial state. $x y=00$ always sets the internal state to $c=0$, while $x y=11$ always sets $c$ to 1 . The other two $x y$ values leave the internal state unchanged. Hence, $x y=00$ and 11 automatically correct an erroneous state of SA; the other two input vectors do not.

It follows that a transition from either of SA's two internal states has probability $1 / 2$. Once returned to a good state, SA operates correctly until a new fault occurs. This leads to the Markov model shown in Fig. 4b. If all four input combinations $x y$ are equiprobable, the probability of remaining in a bad (erroneous) state $k$ cycles after entering a bad state is $0.5^{k}$. The circuit is thus $\left(k, 1-0.5^{k}\right)$-TET, i.e., it is TET with NEP $k$ and the probability of self-recovery $1-0.5^{k}$. Fig. 5 shows how the probability $p_{e r r}(k)$ of an error lurking in SA decreases exponentially with time. Thus, we can, in cases like this, derive an analytic formula for $p_{\text {err }}(k)$

TABLE 2

Classification of All STFs Affecting SA of Fig. 3

\begin{tabular}{c|l|r}
$\begin{array}{c}\text { STF } \\
\text { class }\end{array}$ & Class definition & $\begin{array}{r}\text { No. of STFs } \\
\text { in class }\end{array}$ \\
\hline$F_{0}$ & No effect on SA & 40 \\
\hline$F_{1}$ & $\begin{array}{l}\text { Erroneous output } z \text { in cycle } 0 ; \\
\text { no effect on next state } c \text { in cycle 0 }\end{array}$ & 20 \\
\hline$F_{2}$ & $\begin{array}{l}\text { No effect on } z \text { in cycle } 0 ; \\
\text { erroneous } c \text { in cycle } 0\end{array}$ & 12 \\
\hline$F_{3}$ & $\begin{array}{l}\text { Erroneous } z \text { in cycle } 0 ; \\
\text { erroneous } c \text { in cycle 0 }\end{array}$
\end{tabular}

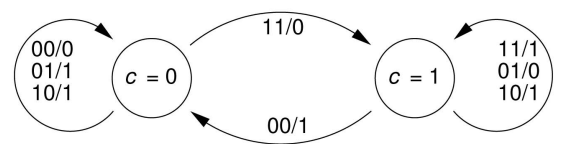

(a)

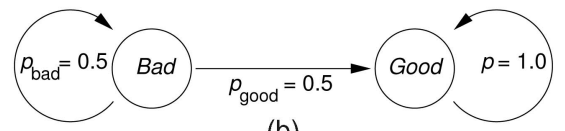

(b)

Fig. 4. (a) State transition graph, and (b) Markov model for SA.

that can be used to determine error tolerance with respect to given thresholds on $p_{\text {err }}(k)$ or $k$.

\subsection{Calculation of $p_{\text {err }}(k)$}

For large or unstructured sequential circuits, computer simulation can be used to determine $p_{\text {err }}(k)$, the probability that the circuit's state is still erroneous after $k$ clock cycles, numerically. The procedure, which reduces the calculation of $p_{e r r}(k)$ to the calculation of $p_{e r r}$ in a combinational circuit (described in Sections 4.2 and 4.3), is outlined next.

First, we construct a combinational $k$ time-frame expansion $T F E_{k}(C)$ of the sequential circuit $C . T F E_{k}(C)$ consists of $k$ copies of $C^{\prime}$ 's combinational core, denoted by $C_{1}, \ldots, C_{k}$. The secondary outputs of $C_{j}$ are connected to the secondary inputs of $C_{j+1}$; the primary inputs (outputs) of $C_{j}$ represent the primary inputs (outputs) of the sequential circuit $C$ in the $j$ th clock cycle. The secondary inputs of $C_{1}$ correspond to the initial state of $C$, and the secondary outputs of $C_{k}$ correspond to $C^{\prime}$ s state after $k$ clock cycles. $T F E_{k}(C)$ is employed in automatic test pattern generation for sequential circuits as follows: the behavior of a circuit affected by a

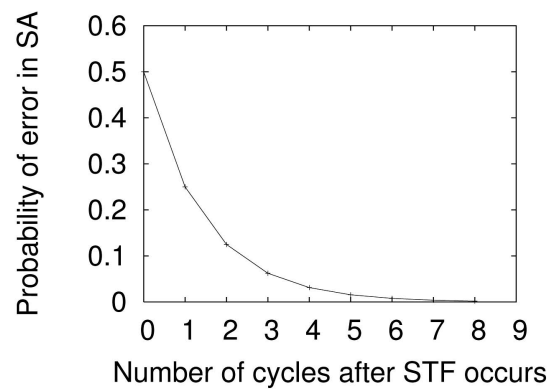

(a)

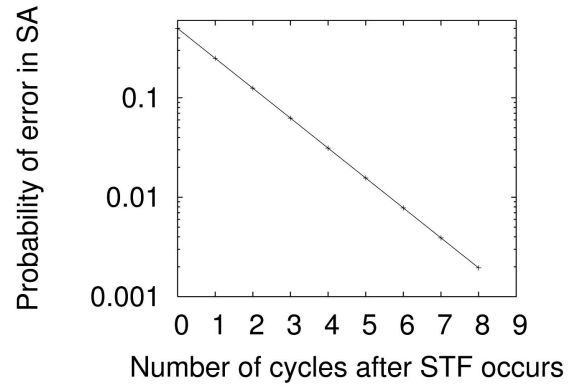

(b)

Fig. 5. Probability $p_{\text {err }}(k)$ of an error in SA's state $k$ cycles after an STF shown (a) in normal form and (b) in logscale form. 
permanent fault, e.g., a stuck-at fault, is obtained by injecting the fault into each $C_{j}$ [1].

Second, $p_{\text {err }}(k)$ is calculated by determining the detection probabilities of the stuck-at faults in circuit $T F E_{k}(C)$ using either the exact method from (8) or the heuristic from Section 4.3 and applying (7). There are two important restrictions as follows: first, only single-stuck-at faults in the first time frame, i.e., $C_{1}$, are considered. These faults represent a disturbance which occurs in the first clock cycle and disappears later, which corresponds to the STF model. (In contrast, permanent faults considered during sequential test generation are injected into all $C_{j}$ s, leading to multiple faults.) Second, only the secondary outputs of $C_{k}$ are taken into account (7), because they represent the circuit's state after $k$ clock cycles.

With these restrictions, $p_{\text {err }}$ of $T F E_{k}(C)$ yields $p_{\text {err }}(k)$. The probability of self-recovery within $k$ cycles $p_{s r}(k)$ is obtained as $1-p_{\text {err }}(k)$. Note that $p_{\text {err }}(k)$ is different from $P^{c}\left(S F \mid F F_{i}\right)$ studied in [4]. $P^{c}\left(S F \mid F F_{i}\right)$ is the probability that a soft error in flip-flop $F F_{i}$ leads to an error at a primary output in at least one clock cycle within $c$ clock cycles after the soft error has occurred. $P^{c}\left(S F \mid F F_{i}\right)$ grows with increasing number of cycles $c$, often reaching values close to 1 , while $p_{\operatorname{err}(k)}$ declines with increasing number of cycles $k$.

\section{Critical Soft Error Rate}

The soft error rate (SER) is the frequency with which soft errors occur. The CSER describes the rate of errors that can lead to serious disruptions of circuit operation, while the SER describes the frequency of bit-flips in the circuit, some of which do not propagate anywhere. Hence, the CSER appears to be the more useful indicator of the circuit's susceptibility to soft errors. Based on this assumption, we study the relationship between CSER and SER, as well as ways to minimize the CSER (rather than the SER) by selective hardening.

Let $e_{1}, e_{2}, \ldots, e_{n}$ be different soft errors that potentially affect circuit operation according to a soft error model. Possible error models include the STF model introduced earlier, STF enriched by electrical information, and aggregated models where one error is composed of multiple STFs. The SER of an individual error and that of the whole circuit are introduced first. Let the SER of an error $e$, $S E R(e)$, denote the probability that the error occurs, i.e., a logical value on a circuit node flips. Note that no propagation of the error to a primary output or a memory element is required. Let the SER of the circuit be the sum of the SERs of the individual errors as follows:

$$
S E R=\sum_{i=1}^{n} \operatorname{SER}\left(e_{i}\right) .
$$

This assumes that soft errors are independent stochastic events (as pointed out above, this assumption is justified in current and future CMOS technologies where soft errors are few and far between). The actual SER is determined by the physical parameters of the circuit and its manufacturing technology. Calculation of SER taking physical parameters into account is beyond the scope of this paper; a large body of literature exists on this subject [7], [12], [29], [39].

Soft errors that are masked and have no effect on the outputs of the circuits are always noncritical, such as the errors in the class $F_{0}$ of the serial adder. If the masking probability of an error $e_{i}$ is $p_{m a s k, i}$ and all errors that are not masked are assumed to be critical, then the CSER is given by

$$
C S E R=\sum_{i=1}^{n} \operatorname{SER}\left(e_{i}\right) \cdot\left(1-p_{\text {mask }, i}\right) .
$$

If the value $p_{\text {mask }, i}$ is calculated taking only logical masking into consideration, then the error probability $p_{\text {err }}$ introduced above can be expressed:

$$
p_{\text {err }}=C S E R / S E R \text {. }
$$

It is possible to take into account further masking mechanisms defined by low-level error models.

Observe that the error rate actually measured on the circuit's outputs, e.g., in an accelerated testing experiment using a radiation source, is CSER rather than SER according to our definitions, because masking is already accounted for in such an experimental setup. In this paper, SER is the error rate which can be derived from the individual error rates of the components as indicated (9). This simplifies the extension of CSER into the framework of transient-error tolerance, as described below.

Suppose that an error effect is defined as noncritical if it does not affect the state of the circuit after $k$ cycles where $k$ is the NEP. Let $p_{\text {mask }, i}(k)$ be the probability that the effect of error $e_{i}$ does not propagate to the circuit state after $k$ cycles. Since we study values of $k$ which are far smaller than the likely number (trillions) of clock cycles between two soft errors, we can safely assume that a second soft error will not occur within $k$ cycles.

While the SER of the circuit is not modified by such a loosening of the specification, the CSER changes. We define

$$
\operatorname{CSER}(k)=\sum_{i=1}^{n} \operatorname{SER}\left(e_{i}\right) \cdot\left(1-p_{\text {mask }, i}(k)\right),
$$

and observe that for $p_{e r r}(k)$, the probability that the circuit is still affected by an error after $k$ cycles, the following relationship similar to (11) holds:

$$
p_{\text {err }}(k)=C S E R(k) / S E R \text {. }
$$

\section{Selective Hardening}

The goal of any hardening strategy is to create a circuit that meets some SER or CSER objectives. We assume that individual circuit nodes can be hardened, thus reducing their SER contribution (9) and improving the overall SER and CSER. Selective hardening has been investigated in [22], [25], [33]. ${ }^{1}$ While the actual hardening mechanism is out of this paper's scope, we mention several approaches from the literature. In a study by NXP [25], logic gates to be hardened were simply duplicated, leading to SER improvement by 60 percent at 20 percent cost. Garg et al. [10] supplemented the duplication by connecting the outputs of the gates by a diode or a transistor. There are also techniques to harden the flip-flops of the circuit, or a subset of its flip-flops [16], [24], [38].

We discuss a minimum-cost selective-hardening strategy to achieve a given CSER target. In contrast to [10], [22], [25],

1. A similar approach in the context of error detection is found in [13]. 
we formulate the selection of the nodes to be hardened as a general optimization problem. We first summarize our assumptions on the selective hardening mechanism. Then, we discuss possible optimization criteria and solution approaches for both CSER targets (no error effect is allowed to show up on the outputs) and relaxed $C S E R(k)$ targets (an error is required to be removed from the system after $k$ cycles). We conclude with an example using the serial adder introduced above.

\subsection{Selective Hardening Mechanism}

Let the set of STFs $f(l / p, x, s)$ with identical $l$ and $p$ be called an error $e_{l / p}$. An error $e_{l / p}$ can be regarded as a stuck-at$p$ fault on the line $l$ that persists for only one clock cycle. The number of such errors, $n$, equals $|S A F|$. Hardening to eliminate or mask the effect of an STF $f(l / p, x, s)$ at a circuit node $l$ masks all the STFs included in the corresponding error $e_{l / p}$, and for brevity, we say that $e_{l / p}$ is hardened. In order to determine which fault sites should be hardened, we consider the set of all errors $e_{l / p}$, denoted by $e_{1}, e_{2}, \ldots, e_{n}$, and determine a subset of the errors to harden so as to meet the desired specification for SER. We assume that any subset of the errors $e_{1}, e_{2}, \ldots, e_{n}$ can be selected for hardening.

If error $e_{i}$ is selected for hardening, $\operatorname{cost} c_{i}$ is incurred and the susceptibility of the circuit to error $e_{i}$ changes from $\operatorname{SER}\left(e_{i}\right)$ to $s_{i} \cdot \operatorname{SER}\left(e_{i}\right)$, where $0 \leq s_{i}<1$. The actual costs of hardening are determined by the particular technique used. For instance, gate duplication [10], [25] would increase the gate count by the number of gates hardened. Note that this increase may not translate into an area increase due to potential routing overhead. Moreover, the duplicated gates are more expensive in [10] than in [25]. There could also be some optimization potential from sharing the duplicated logic. Using BISER [38] as the hardening mechanism for a flip-flop would lead to little area but significant energy overhead. An accurate model of hardening costs $c_{i}$ is not in the scope of this paper. In our experiments, we assume that the hardening costs are proportional to the number of gates to be hardened (by setting all $c_{i}$ s to 1 ).

If errors $e_{i_{1}}, e_{i_{2}}, \ldots, e_{i_{m}}, m \leq n$, have been selected for hardening, the SER of the selectively hardened circuit becomes

$$
S E R_{s h}=\sum_{j=1}^{m} s_{i_{j}} \cdot S E R\left(e_{i_{j}}\right)+\sum_{i \notin\left\{i_{1}, i_{2}, \ldots, i_{m}\right\}} S E R\left(e_{i}\right) .
$$

Equivalently, $s_{i}$ can be set to 1 for all errors $e_{i}$ not selected for hardening $\left(i \notin\left\{i_{1}, i_{2}, \ldots, i_{m}\right\}\right)$. Then

$$
S E R_{s h}=\sum_{i=1}^{n} s_{i} \cdot \operatorname{SER}\left(e_{i}\right) .
$$

If all errors visible at the outputs are considered critical, the CSER becomes

$$
C S E R_{s h}=\sum_{i=1}^{n} s_{i} \cdot \operatorname{SER}\left(e_{i}\right) \cdot\left(1-p_{\text {mask }, i}\right) .
$$

If errors that are flushed out of the circuit state after $k$ cycles can be tolerated, the CSER is given by

$$
C S E R_{s h}(k)=\sum_{i=1}^{n} s_{i} \cdot S E R\left(e_{i}\right) \cdot\left(1-p_{m a s k, i}(k)\right) .
$$

The derating factor $D$ is the SER of the nonhardened circuit divided by the critical soft error rate of the selectively hardened circuit as follows:

$$
\begin{aligned}
D & =S E R / C S E R_{s h}, \\
D(k) & =S E R / C S E R_{s h}(k) .
\end{aligned}
$$

The derating factor indicates the combined improvement from the selective hardening and the consideration of only the critical errors instead of all errors. When no hardening is done and the SER contributions of individual nodes are equal, $D=1 / p_{\text {err }}$. If the "raw" SER of the circuit is known, e.g., from accelerated testing of circuit elements, the derating factor can be used to calculate the CSER of the hardened design, which is the relevant characteristic of the circuit as argued earlier.

The costs $c_{i}$ may be derived from the extra area or power consumption associated with hardening. We assume that these costs are additive, i.e., hardening the circuit against a subset of errors has a cost equal to the sum of the costs $c_{i}$ corresponding to the individual errors.

\subsection{Selecting Nodes for Hardening}

One possible goal of selective hardening is to achieve a given level of CSER at lowest cost by selecting an optimal subset of errors for hardening. In other words, given a desired CSER threshold $T h_{C S E R}$, select a subset of errors for hardening such that $C S E R$, as defined (16), does not exceed the threshold, and the sum of the costs is minimal. Alternatively, a derating threshold $T h_{D}$ may be given. Then, the derating factor $D$ must exceed the derating threshold $T h_{D}$, while the costs are minimized. The same criteria can be set for $C S E R(k)$ and $D(k)$.

Equations (7) and (11) are equivalent to

$$
C S E R=\frac{\sum_{i=1}^{n} S E R\left(e_{i}\right) \cdot D P\left(e_{i}\right)}{|S A F|} .
$$

Hardening a circuit against an individual error $e_{i}$ corresponds to replacing $S E R\left(e_{i}\right)$ by $s_{i} \cdot S E R\left(e_{i}\right)$ in the numerator. Assuming that $s_{i}=0$, i.e., hardening against a soft error eliminates the possibility of error altogether, the corresponding detection probability $D P\left(e_{i}\right)$ can be removed from the numerator. It is possible to sort the errors $e_{i}$ according to $D P\left(e_{i}\right)$ adjusted by the costs of hardening $c_{i}$ and to harden the circuit against the errors in the sorted list until the CSER or the derating target is achieved.

The definition of $p_{e r r}$, and thus derating, focuses on logical masking, and does not take temporal or electrical masking into account. A recent study [40] evaluated the validity of node selection based on logical masking information only (as done in this work), using an accurate electrical-level single-event transient simulator based on the novel UGC particle strike model. It turned out that the derating predicted using logical masking only and the derating measured by the accurate simulator tracks reasonably well, provided that the factors $s_{i}$ are accounted for. (Note that the inverse of $s_{i}$, called the local hardening factor or LHF, is used in [40].) An advantage of a method working at the logical level is its applicability in early design steps, when low-level information necessary to quantify temporal and electrical masking is not available. 


\subsection{Example}

Suppose that the serial adder SA must be TET with $k=2$ and $T h_{D}=15$, i.e., the CSER must be at least 15 times less than the SER. This means that the adder must self-recover after two cycles with probability $1-1 / 15 \approx 0.933$ or more. From Fig. 5, we see that $p_{\text {err }}(2)=0.125$, i.e., $D(2)=8$, which means that $S A$ does not meet the specification and requires hardening. Calculation of the detection probabilities for SAFs is illustrated by the stuck-at- 0 fault on line $x$, denoted by $x / 0$. For this fault, there are four initial state/input sequences which result in an erroneous state after $k=2$ cycles: $s x_{1} y_{1} x_{2} y_{2}=01110,01101,11010$, and 11001. Consequently, the test set size in (8) is four and the detection probability $D P(x / 0)$ is $4 / 2^{5}=0.125$. Similarly, the detection probability also equals 0.125 for faults $x / 1, y / 0, y / 1, s / 0$, and $s / 1$; it is 0.25 for faults $c_{i n} / 0$ and $c_{i n} / 1$; and it is zero for faults $z / 0$ and $z / 1$. For simplicity, we assume that all $s_{i}$ s are 0 and all $c_{i}$ s are 1, i.e., the hardening against any of the errors is associated with identical costs and eliminates any possibility that the error occurs.

A fault with the largest detection probability is selected first, e.g., $c_{i n} / 0$. This reduces $p_{\text {err }}(2)$ by $D P\left(c_{i n} / 0\right) /|S A F|=$ 0.025 , i.e., from 0.125 to 0.1 . The derating is $1 / 0.1=10$, which is still less than 15 . Fault $c_{i n} / 1$, which is selected next, results in $p_{\text {err }}(2)=0.075$ and $D(2) \approx 13.3<15$. Selecting a third fault such as $x / 0$ results in $p_{\text {err }}(2)=0.0625$ and $D(2)=16$, which is above $T h_{D}$. The specification has been met by hardening node $c$ against both flip-to- 0 and flip-to- 1 errors and hardening node $x$ against flip-to-0 errors only. The hardening cost is three or 30 percent of the cost of hardening all nodes (which is 10). Note that by selecting only the most critical nodes to harden, the achieved reduction of 50 percent for $p_{\text {err }}(2)$ exceeds the proportion of hardened nodes (30 percent).

In cases where a Markov model can be constructed, such as SA, it is not necessary to consider all $k$ cycles explicitly. Since we know that for SA, $p_{\text {err }}(2)=p_{\text {err }} \cdot(0.5)^{2}$ (or, equivalently, $\left.D(2)=D /(0.5)^{2}\right)$ holds, it suffices to select faults considering only the probability that the circuit enters state Bad (in Fig. 4b) in the beginning. To meet the specification, this probability must be below $1 /\left(T h_{D} \cdot 0.5^{2}\right) \approx 0.266$, while the actual probability is 0.5 . It is easy to see that by selecting faults $c_{i n} / 0, c_{i n} / 1$, and $x / 0$, the probability becomes 0.25 . A significant reduction in computational complexity is thus achieved with no loss of accuracy. Hence, it is preferable to construct Markov models of the target circuits where feasible.

\subsection{Node Selection Using Approximate Information}

The method outlined in Section 7.2 can employ approximate detection probabilities calculated using the method from Section 4.3. In this case, the value of CSER or derating achieved so far may be erroneously qualified as satisfying the target, i.e., exceeding the threshold, while the actual CSER or derating (calculated using exact information) is below the threshold.

To compensate for this possible overestimation of the solution quality, we set an optimistic derating or CSER target for the approximate method by multiplying the threshold with a safety margin $S M \geq 1$. For instance, for a derating threshold of 10 and $S M=2$, we terminate node selection when the derating (calculated using approximate information) exceeds $20=10 \cdot 2$. The influence of $S M$ on the robustness of the results is discussed in Section 8.2.
TABLE 3

$p_{\text {err }}$ and Cost of Selective Hardening for the ISCAS-85 Combinational Benchmarks

\begin{tabular}{l|r|rr|rr|rr|rr} 
Cct. & $p_{\text {err }}$ & \multicolumn{2}{|c|}{$T h_{D}=10$} & \multicolumn{2}{|c}{$T h_{D}=100$} & \multicolumn{2}{c}{$T h_{D}=1000$} & \multicolumn{2}{c}{$T h_{D}=10000$} \\
& & Cost & $\%$ & Cost & $\%$ & Cost & $\%$ & Cost & $\%$ \\
\hline c17 & 0.299 & 16 & 47.06 & 34 & 100 & 34 & 100 & 34 & 100 \\
c432 & 0.105 & 5 & 0.58 & 557 & 64.47 & 752 & 87.04 & 827 & 95.72 \\
c499 & 0.198 & 158 & 15.83 & 563 & 56.41 & 847 & 84.87 & 965 & 96.69 \\
c880 & 0.198 & 317 & 18.01 & 1130 & 64.20 & 1498 & 85.11 & 1648 & 93.64 \\
c1355 & 0.152 & 246 & 9.08 & 1622 & 59.85 & 2197 & 81.07 & 2564 & 94.61 \\
c1908 & 0.185 & 672 & 17.61 & 1963 & 51.44 & 2685 & 70.36 & 3430 & 89.88 \\
c2670 & 0.167 & 675 & 12.64 & 2899 & 54.29 & 3883 & 72.72 & 4314 & 80.79 \\
c3540 & 0.127 & 332 & 4.69 & 3300 & 46.61 & 5372 & 75.88 & 6388 & 90.23 \\
c5315 & 0.135 & 676 & 6.36 & 6750 & 63.50 & 9537 & 89.72 & 10241 & 96.34
\end{tabular}

\section{Experimental Results}

Next, we describe an application of the foregoing methodology to the mitigation of soft errors in combinational and sequential circuits.

\subsection{Combinational Circuits}

Using symbolic simulation, we calculated the effect of selective hardening on $p_{\text {err }}$ for nine combinational ISCAS85 benchmark circuits for which BDD-based simulation was feasible. Table 3 gives $p_{\text {err }}$ (with $k=0$ ) for a circuit with no hardening and, for four values of the derating threshold $T h_{D}$, the cost of selective hardening to achieve $\left(1 / p_{\text {err }}\right) \geq T h_{D}$, assuming $s_{i}=0$ and $c_{i}=\operatorname{SER}\left(e_{i}\right)=1$ for all errors. (The overall cost of the hardening corresponds to the number of stuck-at faults excluded from (20) and the percentage of these faults among all faults.) The detection probability in the numerator of (20) has been obtained using (7). For this purpose, we constructed, for each stuck-at fault, BDDs for the outputs of the fault-free and faulty circuits using the CUDD package and applied BDD operations provided by that package to calculate the test set and its size. We employed arbitrary precision arithmetic to represent large numbers. Recall that every stuck-at fault represents a class of STFs in this analysis.

The probability $p_{\text {err }}$ of a soft error showing up on an output is between 0.1 and 0.2 for all circuits except c17, for which it is 0.3 . This means that only approximately every fifth to tenth soft error is actually visible on a circuit output and the other faults are masked by the circuit itself. These findings are consistent with the data in [22], [25], even though the modeling in these works took low-level information into account. The cost of selective hardening with a derating factor of 10 is generally quite low. In contrast, higher derating thresholds require overheads which are probably unacceptable. Recall that no high derating factors may be required for combinational circuits as the faulty effect will definitely last only for one clock cycle. Hence, selective hardening is useful for combinational circuits if the derating threshold is not much larger than the actual derating figure, which is likely to be the case.

\subsection{Approximate Method}

We applied the node selection algorithm based on approximate detection probabilities to the combinational parts of the ISCAS-89 circuits. We used a derating threshold of 10 and three different safety margins. Fig. 6 shows the actual derating (computed using exact information) of the solutions found. One can see that the approximate algorithm indeed 


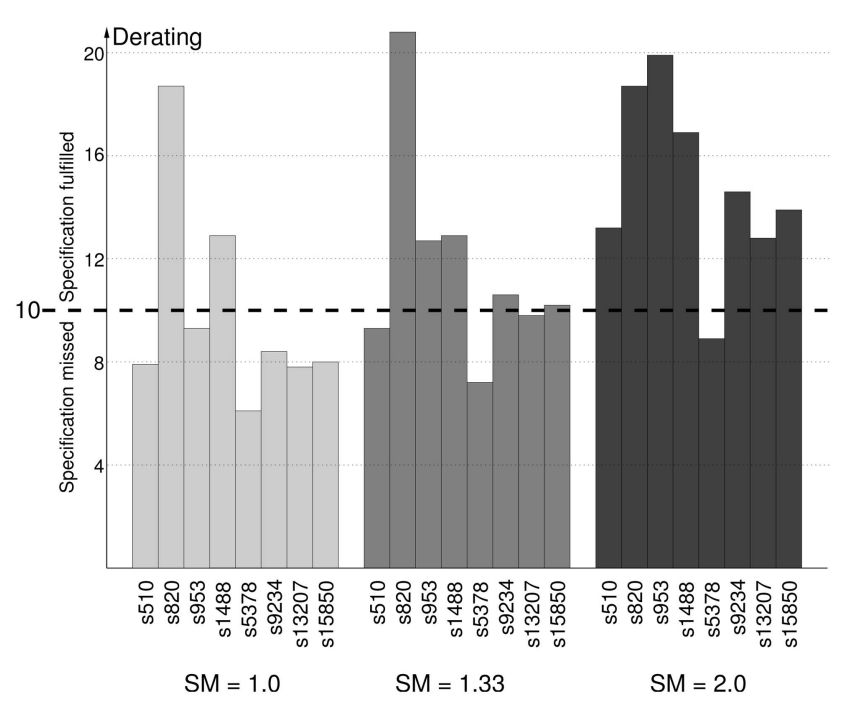

Fig. 6. Exact derating achieved by the approximate method with derating target 10 and safety margins $1,1.33$, and 2 .

tends to overestimate the derating, resulting in an early termination of node selection, and thus, a solution of insufficient quality. Employing a safety margin of two, the target is met for almost all cases (the method is heuristic and cannot be expected to perform as well as the exact algorithm). It is advisable to use a safety margin of this order of magnitude for large circuits for which the BDDs cannot be generated, and thus, no exact derating figures are available.

To evaluate the scalability of the approximate method, we applied it to the combinational cores of some large industrial circuits provided by NXP. The results for node selection with derating target $D^{\text {target }}=10$ and $D^{\text {target }}=20$ are reported in Table 4. Column \% contains the percentage of the selected soft errors (given in the column $H$ ) among all soft errors. The exact method could not be run for these circuits because of the memory requirements of the BDDs. The total number of soft errors again equals twice the number of gates in the circuit, roughly indicated by the circuit names. The runtimes are in CPU seconds and do not include the time to load the circuit (which took around $15 \mathrm{~s}$ for the largest circuit p2927k with approximately 2.5 million gates).

The results suggest that high derating values can be obtained for large circuits with a reasonable overhead and that the method is scalable.

TABLE 4

Selective Hardening of Industrial Circuits with Derating Target

\begin{tabular}{lr|rrr|rrr} 
Circuit & Soft & \multicolumn{3}{|c|}{$T h_{D}=10$} & \multicolumn{3}{|c}{$T h_{D}=20$} \\
& errors & $H$ & $\%$ & Time & $H$ & $\%$ & Time \\
\hline p35k & 93456 & 6965 & 7.45 & 0.05 & 16605 & 17.77 & 0.05 \\
p45k & 87442 & 10713 & 12.25 & 0.05 & 20301 & 23.22 & 0.06 \\
p77k & 143266 & 7706 & 5.38 & 0.10 & 17394 & 12.14 & 0.09 \\
p78k & 154782 & 8218 & 5.31 & 0.10 & 28270 & 18.26 & 0.10 \\
p81k & 185674 & 22545 & 12.14 & 0.13 & 51191 & 27.57 & 0.13 \\
p100k & 193338 & 27683 & 14.32 & 0.12 & 53254 & 27.54 & 0.13 \\
p267k & 559650 & 116334 & 20.79 & 0.41 & 188020 & 33.6 & 0.41 \\
p330k & 696048 & 54346 & 7.81 & 0.53 & 132966 & 19.1 & 0.54 \\
p378k & 773894 & 41082 & 5.31 & 0.56 & 141335 & 18.26 & 0.57 \\
p2927k & 4887944 & 502711 & 10.28 & 4.20 & 1105949 & 22.63 & 4.31
\end{tabular}

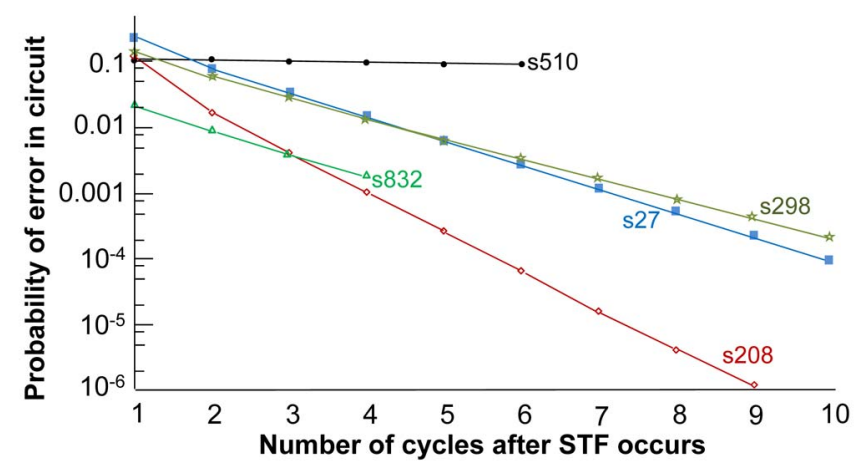

Fig. 7. $p_{\text {err }}(k)$ for ISCAS-89 circuits in graph form (logscale).

\subsection{Sequential Circuits}

Fig. 7 shows the values of $p_{\text {err }}(k)$ for the sequential ISCAS- 89 circuits and various values of $k$ in graph form. Note that $p_{e r r}(k)$ is the probability that the error still affects the circuit state after $k$ cycles and that it decreases with $k$. It can be seen that for $k>1$, the graphs closely approximate straight lines. This means that the ratio $p_{\text {err }}(k+1) / p_{\text {err }}(k)$ is nearly constant for a given circuit, although it does vary significantly from circuit to circuit as demonstrated by the slope of the curves.

The data from Fig. 7 suggest that knowing $p_{\text {err }}(k)$ for $k=1,2$, and 3 is sufficient for an accurate analysis in most cases, and it is possible to approximate $p_{\text {err }}(k)$ for larger values of $k$ as follows:

$$
p_{\text {err }}(k) \approx p_{\text {err }}(2) \cdot\left(p_{\text {err }}(3) / p_{\text {err }}(2)\right)^{k-2} \quad(k>2) .
$$

It is also possible to construct a Markov model similar to that in Fig. 4 based on the probabilities $p_{\text {err }}(1), p_{\text {err }}(2)$, and $p_{e r r}(3)$. Note that this approximation is orthogonal to the detection-probability-based approximated node selection from Section 7.4.

TET and selective hardening are evaluated in Table 5 for the ISCAS- 89 benchmark circuit s298. The table shows the number of gates to be hardened and their percentage to meet four alternative derating targets. It can be seen that selective hardening is indeed a low-cost way to reach a given derating threshold if nonreference behavior is acceptable for a few clock cycles. For example, improvement by three orders of magnitude is possible by hardening just seven percent of nodes if the noncritical error period is set to seven.

TABLE 5

Selective Hardening of Circuit s298

\begin{tabular}{r|rr|rr|rr|rr}
$k$ & \multicolumn{2}{|c}{$T h_{D}=10$} & $T h_{D}=100$ & \multicolumn{2}{c}{$T h_{D}=1000$} & \multicolumn{2}{c}{$T h_{D}=10000$} \\
& Cost & $\%$ & Cost & $\%$ & Cost & $\%$ & Cost & $\%$ \\
\hline 1 & 50 & 8.39 & 351 & 58.89 & 508 & 85.23 & 553 & 92.79 \\
2 & 0 & 0 & 186 & 31.21 & 403 & 67.62 & 492 & 82.55 \\
3 & 0 & 0 & 99 & 16.61 & 293 & 49.16 & 457 & 76.68 \\
4 & 0 & 0 & 23 & 3.86 & 188 & 31.54 & 368 & 61.74 \\
5 & 0 & 0 & 0 & 0 & 146 & 24.50 & 252 & 42.28 \\
6 & 0 & 0 & 0 & 0 & 102 & 17.11 & 208 & 34.90 \\
7 & 0 & 0 & 0 & 0 & 42 & 7.05 & 182 & 30.54 \\
8 & 0 & 0 & 0 & 0 & 0 & 0 & 155 & 26.01 \\
9 & 0 & 0 & 0 & 0 & 0 & 0 & 116 & 19.46 \\
10 & 0 & 0 & 0 & 0 & 0 & 0 & 67 & 11.24 \\
11 & 0 & 0 & 0 & 0 & 0 & 0 & 5 & 0.84
\end{tabular}




\section{Conclusions}

We introduced a general framework to model transient errors taking their impact on circuit functionality into account, in particular, their ability to disturb the circuit state for specified periods of time. Using the STF model, we defined various practical and technology-independent metrics for the errors of interest. These metrics include the error probability $p_{e r r}$ for an individual circuit node, and the probability of self-recovery $p_{s r}$ for the entire circuit. We also introduced the critical soft error rate CSER and the derating factor $D$, which can serve as measures of soft error susceptibility/tolerance of a design during logic synthesis. All these metrics can be computed quite efficiently using conventional simulation and ATPG techniques and, in the case of very large circuits, can be approximated quickly. We demonstrated the successful application of the proposed methodology in the case of the ISCAS- 89 benchmarks achieving significant CSER improvement at limited cost.

\section{ACKNOWLEDGMENTS}

This work was supported in part by the Alexander Von Humboldt Foundation, the DFG Project RealTest under Grants BE 1176/15-2 and PO 1220/2-2, and the US Air Force Research Laboratory under Agreement No. FA8750-05-10282. We are thankful to J. Schlöffel of NXP for providing the industrial benchmarks. Parts of this paper are based on "An Analysis Framework for Transient-Error Tolerance" which was presented at the VLSI Test Symposium, May 2007 [11] and on "Scalable Calculation of Logical Masking Effects for Selective Hardening against Soft Errors" which was presented at the International Symposium on VLSI, April 2008 [30].

\section{REFERENCES}

[1] M. Abramovici, M.A. Breuer, and A.D. Friedman, Digital Systems Testing and Testable Design. Computer Science Press, 1990.

[2] S. Almukhaizim, T. Verdel, and Y. Makris, "Cost-Effective Graceful Degradation in Speculative Processor Subsystems: The Branch Prediction Case," Proc. IEEE Int'l Conf. Computer Design, pp. 194-197, 2003.

[3] H. Ando, R. Kan, Y. Tosaka, K. Takahisa, and K. Hatanaka, "Validation of Hardware Error Recovery Mechanisms for the SPARC64 V Microprocessor," Proc. Int'l Conf. Dependable Systems and Networks, pp. 62-69, 2008.

[4] H. Asadi and M. Tahoori, "Soft Error Modeling and Protection for Sequential Elements," Proc. IEEE Defect and Fault Tolerance Symp., pp. 463-471, 2005.

[5] M. Breuer and H. Zhu, "An Illustrated Methodology for Analysis of Error Tolerance," IEEE Design and Test of Computers, vol. 25, no. 2, pp. 168-177, Mar./Apr. 2008.

[6] M.A. Breuer, "Testing for Intermittent Faults in Digital Circuits," IEEE Trans. Computers, vol. 22, no. 3, pp. 241-246, Mar. 1973.

[7] P.E. Dodd and L.W. Massengill, "Basic Mechanisms and Modeling of Single-Event Upset in Digital Microelectronics," IEEE Trans. Nuclear Science, vol. 50, no. 3, pp. 583-602, June 2003.

[8] K. Driscoll, B. Hall, H. Sivencrona, and P. Zumsteg, "Byzantine Fault Tolerance, from Theory to Reality," Proc. Int'l Conf. Computer Safety, Reliability and Security, pp. 235-248, 2003.

[9] E.N. Elnozahy, L. Alvisi, Y.M. Wang, and D.B. Johnson, "A Survey of Rollback-Recovery Protocols in Message-Passing Systems," ACM Computing Surveys, vol. 34, no. 3, pp. 375-408, 2002.

[10] R. Garg, N. Jayakumar, S.P. Khatri, and G. Choi, "A Design Approach for Radiation-Hard Digital Electronics," Proc. IEEE Design Automation Conf., pp. 773-778, 2006.
[11] J.P. Hayes, I. Polian, and B. Becker, "An Analysis Framework for Transient-Error Tolerance," Proc. Very Large-Scale Integration Test Symp., pp. 249-255, 2007.

[12] S. Hellebrand, C.G. Zoellin, H.-J. Wunderlich, S. Ludwig, T. Coym, and B. Straube, "A Refined Electrical Model for Particle Strikes and Its Impact on SEU Prediction," Proc. IEEE Defect and Fault Tolerance Symp., 2007.

[13] E. Hill, M. Lipasti, and K. Saluja, "An Accurate Flip-Flop Selection Technique for Reducing Logic SER," Proc. Int'l Conf. Dependable Systems and Networks, pp. 32-41, 2008.

[14] S.K. Jain and V.D. Agrawal, "Statistical Fault Analysis," IEEE Design and Test of Computers, vol. 2, no. 1, pp. 38-44, Jan./Feb. 1985.

[15] Z. Jiang and S. Gupta, "Threshold Testing: Improving Yield for Nanoscale VLSI," IEEE Trans. Computer-Aided Design of Integrated Circuits and Systems, vol. 28, no. 12, pp. 1993-1895, Dec. 2009.

[16] V. Joshi, R.R. Rao, D. Blaauw, and D. Sylvester, "Logic SER Reduction through Flip Flop Redesign," Proc. Int'l Symp. Quality Electronic Design, pp. 611-616, 2006.

[17] S. Krishnaswamy, G.F. Viamontes, I.L. Markov, and J.P. Hayes, "Probabilistic Transfer Matrices in Symbolic Reliability Analysis of Logic Circuits," ACM Trans. Design Automation of Electronic Systems, vol. 13, no. 1, 2008.

[18] W.Y. Kung, C.S. Kim, and C.C.J. Kuo, "Spatial and Temporal Error Concealment Techniques for Video Transmission over Noisy Channels," IEEE Trans. Circuits and Systems for Video Technology, vol. 16, no. 7, pp. 789-802, July 2006.

[19] X. Li and D. Yeung, "Application-Level Correctness and Its Impact on Fault Tolerance," Proc. Int'l Symp. High Performance Computer Architecture, pp. 181-192, 2007.

[20] J.W.S. Liu, W.K. Shin, K.J. Lin, R. Bettati, and J.Y. Chung, "Imprecise Computations," Proc. IEEE, vol. 82, no. 1, pp. 83-94, Jan. 1994

[21] M. May, M. Alles, and N. Wehn, "A Case Study in ReliabilityAware Design: A Resilient LDPC Code Decoder," Proc. Conf. Design, Automation and Test in Europe, 2008.

[22] K. Mohanram and N.A. Touba, "Cost-Effective Approach for Reducing Soft Error Failure Rate in Logic Circuits," Proc. IEEE Int'l Test Conf., pp. 893-901, 2003.

[23] H.T. Nguyen and Y. Yagil, "A Systematic Approach to SER Estimation and Solutions," Proc. Int'l Reliability Physics Symp., pp. 60-70, 2003

[24] M. Nicolaidis, "GRAAL: A Fault-Tolerant Architecture for Enabling Nanometric Technologies," Proc. Int'l On-Line Test Symp., p. 255, 2007.

[25] A.K. Nieuwland, S. Jasarevic, and G. Jerin, "Combinational Logic Soft Error Analysis and Protection," Proc. Int'l On-Line Test Symp., 2006.

[26] D. Nowroth, I. Polian, and B. Becker, "A Study of Cognitive Resilience in a JPEG Compressor," Proc. Int'l Conf. Dependable Systems and Networks, pp. 32-41, 2008.

[27] Z. Pan and M.A. Breuer, "Basing Acceptable Error-Tolerant Performance on Significance-Based Error-Rate (SBER)," Proc. Very Large-Scale Integration. Test Symp., 2008.

[28] I. Polian, B. Becker, M. Nakasato, S. Ohtake, and H. Fujiwara, "Low-Cost Hardening of Image Processing Applications against Soft Errors," Proc. Int'l Symp. Defect and Fault Tolerance, pp. 274279, 2006.

[29] I. Polian, J.P. Hayes, S. Kundu, and B. Becker, "Transient Fault Characterization in Dynamic Noisy Environments," Proc. IEEE Int'l Test Conf., pp. 1039-1048, 2005.

[30] I. Polian, S.M. Reddy, and B. Becker, "Scalable Calculation of Logical Masking Effects for Selective Hardening against Soft Errors," Proc. IEEE Int'l Symp. Very Large-Scale Integration, pp. 257$262,2008$.

[31] C. Rusu, A. Bougerol, L. Anghel, C. Weulerse, N. Buard, S. Benhammadi, N. Renaud, G. Hubert, F. Wrobel, T. Carriere, and R. Gaillard, "Multiple Event Transient Induced by Nuclear Reactions in CMOS Logic Cells," Proc. Int'l On-Line Test Symp., pp. 137-145, 2007.

[32] J. Savir, "Testing for Single Intermittent Failures in Combinational Circuits by Maximizing the Probability of Fault Detection," IEEE Trans. Computers, vol. 29, no. 5, pp. 410-416, May 1980.

[33] S.A. Seshia, W. Li, and S. Mitra, "Verification-Guided Soft Error Resilience," Proc. Conf. Design, Automation and Test in Europe, 2007.

[34] S. Shahidi and S.K. Gupta, "ERTG: A Test Generator for ErrorRate Testing," Proc. IEEE Int'l Test Conf., 2007. 
[35] P. Shivakumar, M. Kistler, W. Keckler, D. Burger, and L. Alvisi, "Modeling the Effect of Technology Trends on the Soft Error Rate of Combinational Logic," Proc. Int'l Conf. Dependable Systems and Networks, pp. 389-398, 2002.

[36] F. Wang and V. Agrawal, "Soft Error Rate Determination for Nanometer CMOS VLSI logic," Proc. Southeastern Symp. System Theory, pp. 324-328, 2008.

[37] H.J. Wunderlich, "PROTEST: A Tool for Probabilistic Testability Analysis," Proc. IEEE Design Automation Conf., 1985.

[38] M. Zhang, S. Mitra, T.M. Mak, N. Seifert, N.J. Wang, Q. Shi, K.S. Kim, N.R. Shanbhag, and S.J. Patel, "Sequential Element Design with Built-In Soft Error Resilience," IEEE Trans. Very Large-Scale Integration Systems, vol. 14, no. 12, pp. 1368-1378, Dec. 2006.

[39] M. Zhang and N.R. Shanbhag, "Soft Error-Rate Analysis (SERA) Methodology," IEEE Trans. Computer-Aided Design, vol. 25, no. 10, pp. 2140-2155, Oct. 2006.

[40] C.G. Zoellin, H.-J. Wunderlich, I. Polian, and B. Becker, "Selective Hardening in Early Design Steps," Proc. European Test Symp., pp. 185-190, 2008.

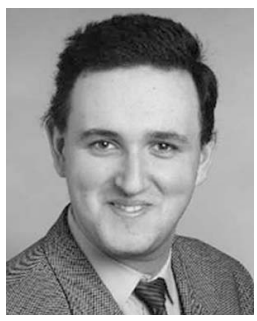

Ilia Polian received the diploma (master's) and $\mathrm{PhD}$ degrees in computer science from the University of Freiburg, Germany, in 1999 and 2003, respectively. He was with Micronas, Freiburg, IBM R\&D, Böblingen, Germany, and the Nara Institute of Science and Technology (NAIST), Japan. He served as a senior member of the scientific staff at the Chair of Computer Architecture at the University of Freiburg and recently joined the University of Passau, Germany, as a full professor of computer engineering. His research interests include defect modeling, design for testability, and formal verification of hybrid and real-time systems. He was a European Champion and Vice World Champion at the 1999 ACM International Collegiate Programming Contest, VDE Award Laureate 1999, and Wolfgang-Gentner Award Laureate 2004. He served as the finance chair of the IEEE European Test Symposium (ETS) 2007, the vice-program chair of the Reliability-Aware System Design and Test Workshop (RASDAT) 2010 and 2011, the General Chair of the GI/ITG/GMM Test Methods and Reliability Workshop 2011, and was on a number of Program Committees. He is a senior member of the IEEE.

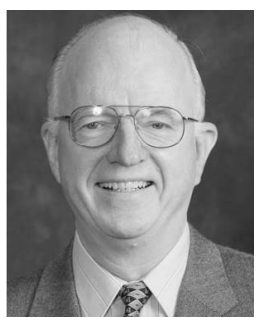

John P. Hayes received the BE degree from the University College Dublin, and the MS and PhD degrees from the University of Illinois, UrbanaChampaign, all in electrical engineering. While at the University of Illinois, he participated in the design of the ILLIAC III computer. He is currently a professor in the Department of Electrical Engineering and Computer Science, University of Michigan, Ann Arbor, where he holds the Claude E. Shannon endowed chair in engineering science. He was previously a faculty member of the Departments of Electrical Engineering Systems and Computer Science at the University of Southern California, Los Angeles. He spent two years with the Operations Research Group at the Shell Benelux Computing Center in The Hague, where he was involved in mathematical programming. He has also held visiting positions at several organizations including Stanford University, LogicVision Inc., and the University of Freiburg. His current teaching and research interests include computer-aided design, verification, and testing; VLSI circuits; computer architecture, especially embedded computer networks; fault-tolerant and safetycritical systems; and quantum computing. He was the founding director of the University of Michigan's Advanced Computer Architecture Laboratory (ACAL). He has authored more than 250 technical papers, several patents, and six books, including Computer Architecture and Organization (third ed., McGraw-Hill, 1998) and Quantum Circuit Simulation (Springer, 2009). He has served as an editor of the Communications of the ACM, the IEEE Transactions on Parallel and Distributed Systems, and the Journal of Electronic Testing. He is a fellow of the IEEE and the ACM, and a member of the Sigma Xi. He received the University of Michigan's Distinguished Faculty Achievement Award in 1999, and the Alexander von Humboldt Foundation's Research Award in 2004.

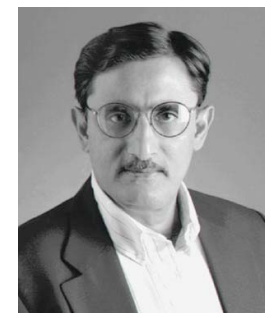

Sudhakar M. Reddy received the BE degree in electronics and communication engineering from Osmania University, Hyderabad, India, the ME degree from the Indian Institute of Science, Bengaluru, India, and the $\mathrm{PhD}$ degree in electrical engineering from the University of lowa. Since 1968, he has been a faculty member in the Department of Electrical and Computer Engineering, University of lowa, where he is currently a University of lowa Foundation distinguished professor. He served as the chair of the department from 1981 to 2000 . He has published more than 500 papers in the areas of test and design for test of digital VLSI circuits, coding theory, and fault-tolerant computing. He is a life fellow of the IEEE. He received a Von Humboldt Senior Research Fellowship in 1995, and a Life Time Achievement Award from VLSI Design Conference. He has served twice as a guest editor and as an associate editor of the IEEE Transactions on Computers, and as an associate editor of the IEEE Transactions on Computer-Aided Design of Integrated Circuits and Systems. He was the technical program chair of the 1989 International Fault-Tolerant Computing Symposium.

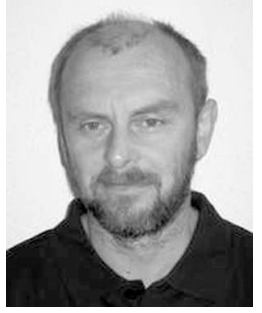

Bernd Becker is a full professor in the Faculty of Engineering, University of Freiburg, Germany. Prior to joining University of Freiburg in 1995, he was with J. W. Goethe-University Frankfurt as an associate professor for complexity theory and efficient algorithms. His research interests include computer-aided design, test, and verification of (digital) circuits and systems (VLSI CAD). A focus of his research is the development and analysis of efficient data structures and algorithms in VLSI CAD. The development of symbolic methods for test and verification of digital circuits and their integration in the industrial flow is one of the major achievements of his work. More recently, he has been working on verification methods for embedded systems and test techniques for nanoelectronic circuitry. He has published more than 200 papers in peer-reviewed conferences and journals and has been on the program and organizing committees of numerous major international conferences. He has been the holder of several research grants from DFG, BMBF, and industry as well. He currently serves as the cospeaker of the DFG transregional collaborative research center "Automatic Analysis and Verification of Complex Systems (AVACS)" with project partners from the University of Freiburg, the University of Saarland, the University of Oldenburg, and the Max Planck Institute of Computer Science. He is a fellow of the IEEE.

$\triangle$ For more information on this or any other computing topic, please visit our Digital Library at www.computer.org/publications/dlib. 